\title{
As Classificações dos Climas das Cidades
}

\author{
Cities Climates Classification
}

\section{Clasificación de los Climas de las Ciudades}

\author{
Larissa Piffer Dorigon ${ }^{1}$ \\ (iD) https://orcid.org/0000-0002-7258-9067
}

Margarete Cristiane de Costa Trindade Amorim²
iD https://orcid.org/0000-0002-3975-493X

RESUMO: A acelerada urbanização brasileira a partir dos anos de 1960 deixou marcas ambientais e sociais nas cidades. Muitos são os indicadores considerados quando se busca avaliar a qualidade ambiental urbana, sendo um deles as ilhas de calor urbanas. Nos estudos sobre esse fenômeno uma etapa considerada basilar é a divisão da cidade em áreas relativamente homogêneas, ou seja, que contenham características minimamente parecidas. Neste sentido, um procedimento que vem sendo utilizado nos estudos de ilhas de calor urbanas é a classificação de unidades climáticas. Sendo assim, este artigo busca discorrer sobre os princípios das classificações climáticas urbanas e aprofundar o conhecimento a respeito da proposta metodológica de Zonas Climáticas Locais (Local Climate Zones - LCZ). Conclui-se que a utilização da metodologia das LCZs possibilita melhor descrição das áreas investigadas, dada a diversidade de características da superfície apresentada no sistema, além de promover a divulgação e a comunicação mais eficiente das análises.

PALAVRAS-CHAVE: Classificação climática. Ilhas de calor urbanas. Clima urbano. Zonas climáticas locais.

ABSTRACT: The accelerated Brazilian urbanization since the 1960s left environmental and social marks in the cities. Many indicators are considered when seeking to assess urban environmental quality, one of which is urban heat islands. In studies on this phenomenon, a step considered basic is the division of the city into relatively homogeneous parts, that is, that contain minimally similar characteristics. In this sense, a procedure that has been used in studies of urban heat islands is the classification of climatic units. Therefore, this article seeks to discuss the principles of urban climate classifications and deepen knowledge about the methodology of Local Climate Zones. It is concluded that the use of the LCZs methodology allows a better description of the investigated areas, given the

\footnotetext{
${ }^{1}$ Doutora em Geografia pelo Programa de Pós-Graduação em Geografia da FCT UNESP, Campus de Presidente Prudente. E-mail: laridorigon@hotmail.com.

2 Doutora em Geografia Física pela USP. Professora do Programa de Pós-Graduação em Geografia da FCT UNESP, Campus de Presidente Prudente. E-mail: margarete.amorim@unesp.br.
} 
diversity of surface characteristics presented in the system, in addition to promoting the dissemination and more efficient communication of analyzes.

KEYWORDS: Climatic classification. Urban heat islands. Urban climate. Local climate Zones.

RESUMEN: La acelerada urbanización brasileña desde la década de 1960 dejó marcas ambientales y sociales em las ciudades. Se consideran muchos indicadores cuando se busca evaluar la calidad ambiental urbana, uno de los cuales son las islas de calor urbano. En los estudios sobre este fenómeno, um paso considerado básico es la división de la ciudad en partes relativamente homogéneas, es decir, que contienen características mínimamente similares. En este sentido, un procedimiento que se ha utilizado en estudios de islas de calor urbanas es la clasificación de unidades climáticas. Por lo tanto, este artículo busca discutir los principios de las clasificaciones del clima urbano y profundizar el conocimiento sobre la metodología de las Zonas Climáticas Locales (Local Climate Zones). Se concluye que el uso de la metodologia LCZs permite una mejor descripción de las áreas investigadas, dada la diversidad de características de la superficie presentada en el sistema, además de promover la difusión y la comunicación más eficientes de los análisis.

PALABRAS CLAVE: Clasificación climática. Islas de calor urbano. Clima urbano. Zonas climáticas locales.

\section{INTRODUÇÃO}

O aumento da urbanização não é uma tendência nova, em 1990, 43\% (2,3 bilhões) da população mundial vivia em áreas urbanas e em 2015 o número cresceu para 54\% (4 bilhões) (ONU-HABITAT, 2016). A previsão é de que até 2030 esse número atinja, aproximadamente, 5 bilhões de pessoas, ou seja, 60\% da humanidade (UNFPA, 2015).

No Brasil, a partir dos anos 1940-1950, a industrialização foi a grande impulsionadora do aumento da população urbana. Neste contexto, ainda conforme Santos (1993), de 1960 a 1980 as cidades brasileiras ganharam cerca de 50 milhões de novos habitantes - número quase similar a população total no país em 1950. Interessante destacar que entre 1980 e 1990 a população brasileira se elevou $26 \%$, enquanto a população urbana (parte da população que vive nas cidades) aumentou mais de $40 \%$. Isso significa afirmar que desde 1960 o Brasil passa por um processo de urbanização (mudança das populações rurais para as cidades - figura 1) atingindo em 2010 a taxa 84,4\%. Deste modo, verifica-se que o crescimento da população urbana no Brasil foi $50,1 \%$ em 60 anos.

Convêm aqui destacar que não somente o número de habitantes confirma a dualidade entre urbano e rural ou até mesmo a expansão da urbanização nacional, uma vez que estes conceitos são ainda bastante discutidos, como exposto no artigo de Chatel e Sposito (2015). No entanto, para os estudos sobre clima urbano, o fator adensamento de construções e as alterações das paisagens com a inserção de materiais artificiais (característicos de cidades) são elementos essenciais para a análise. 
Figura 1 - Gráfico da taxa de urbanização brasileira

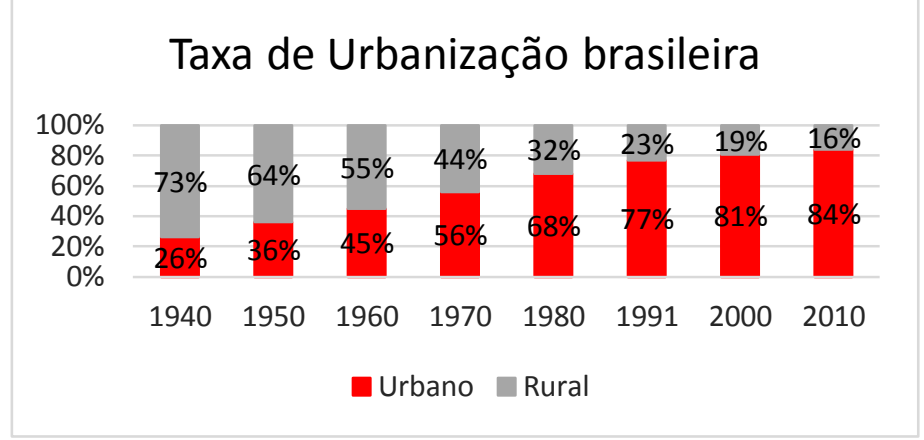

Fonte: IBGE $(2000,2010)$.

Sendo assim, a acelerada urbanização a partir dos anos 1960 deixou marcas ambientais e sociais nas cidades. Muitos são os indicadores considerados quando se busca avaliar a qualidade ambiental urbana, sendo um deles a geração de um clima diferenciado nas cidades, mais especificamente a distribuição das temperaturas nos diferentes espaços intraurbanos em relação ao rural próximo (MONTEIRO, 1976). A importância do estudo desta temática está relacionada com o conforto/desconforto térmico, problemas relacionados à saúde, além de ser entendido como um indicador de exclusão social e espacial (AMORIM et al., 2009).

Aspectos como a rugosidade, a retirada de vegetação arbórea, a densidade de construções e edificações e a geometria do traçado urbano, além das funções econômicas e sociais desenvolvidas no local, podem ser fatores determinantes na geração do clima urbano, uma vez que modificam o balanço energético e hídrico entre a superfície e a atmosfera, afetando, assim, o funcionamento dos demais elementos climáticos (AMORIM, 2000, 2010; ARNFIELD, 2003; MENDONÇA; DUBREUIL, 2005).

Atualmente a resposta climática mais reconhecida causada pela expansão da malha urbana é o fenômeno das ilhas de calor, ou seja, o calor característico de um determinado local em comparação com o seu entorno próximo (AMORIM, 2000, 2017; LIN; YU, 2005; MONTEIRO, 1976; REN; CHU; CHEN; 2007; REN; ZHOU, 2014).

Os materiais utilizados nas construções e a cobertura vegetal, são considerados fatores essenciais na identificação das temperaturas das cidades. De acordo com Grimmond (2007), as propriedades radiativas, térmicas e hidráulicas dos materiais de construção diferem daquelas encontradas no solo, na vegetação e na água e interferem no processo de absorção e liberação de energia e diferenciam a intensidade do aquecimento/arrefecimento.

Uma etapa que pode ser considerada basilar na abordagem das ilhas de calor é a divisão da cidade em partes relativamente homogêneas. Neste sentido, um procedimento que vem sendo utilizado nos estudos de ilhas de calor é a classificação de unidades climáticas. 
Neste sentido, este artigo busca discorrer sobre os princípios das classificações climáticas urbanas e aprofundar o conhecimento a respeito da metodologia elaborada por Stewart e Oke (2009), Zonas Climáticas Locais (Local Climate Zones), na qual cada zona é definida segundo a cobertura de superfície e a estrutura urbana.

\section{AS CLASSIFICAÇÕES DOS CLIMAS DAS CIDADES}

As ilhas de calor, em sua maioria, são aferidas com registros que comparam o ambiente urbano e o rural e a diferença encontrada é considerada na sua intensidade, muitas vezes o fenômeno acaba sendo demasiadamente simplificado, e não corresponde às características paisagísticas dos diferentes ambientes urbanos e rurais.

Stewart (2011) elaborou uma proposta de classificação de uso e ocupação da terra, denominada Local Climate Zones (LCZ) ou Zonas Climáticas Locais, na qual cada zona climática é detalhada de acordo com as propriedades encontradas na superfície (altura da rugosidade superficial, fração de superfície impermeável e desempenho térmico, entre outros) e possibilita ultrapassar a dicotomia "rural x urbano" devido a descrição específica de cada LCZ.

As classificações utilizadas nos estudos de clima urbano se iniciaram em 1965, quando Chandler (1965) classificou a área de Londres em unidades espaciais de acordo com as alturas, densidades e materiais dos edifícios, além da distribuição de vegetação arbórea, parques e espaços abertos (figura 2). Assim, o autor conseguiu sintetizar quatro regiões nas quais as paisagens urbanas eram similares: I - Central; II - Suburbanas Internas; III - Suburbanas Externas e; IV - Altas do Norte (STEWART, 2011).

Auer Jr (1978) propôs um sistema de classificação utilizando critérios de uso da terra e cobertura vegetal e realizou essa classificação para a cidade de St. Louis, nos Estados Unidos. O autor propôs 12 classes na área Metropolitana de St. Louis, das quais 2 (I1 e I2) correspondem ao uso industrial, $1(\mathrm{C} 1)$ ao comércio, 4 (R1, R2, R3 e R4) ao uso residencial e 5 (A1, A2, A3, A4 e A5) a áreas pouco construídas e/ou naturais. Sendo assim, o autor conseguiu, relacionar as anomalias termodinâmicas, cinemáticas e radiativas com as características da superfície de uma forma mais detalhada e específica do que vinha sendo utilizado (centro urbano, comercial, residente).

Já na década de 1990, Ellefsen (1991) elaborou um sistema denominado Urban Terrain Zone (UTZ) para aplicação na escala do intraurbano. O autor, baseado nas características da morfologia das construções, na configuração das ruas e nos materiais utilizados, forneceu um inventário completo com esboços da planta e do perfil de cada zona. 
Figura 2 - Regiões climáticas de Londres de acordo com a classificação de Chandler

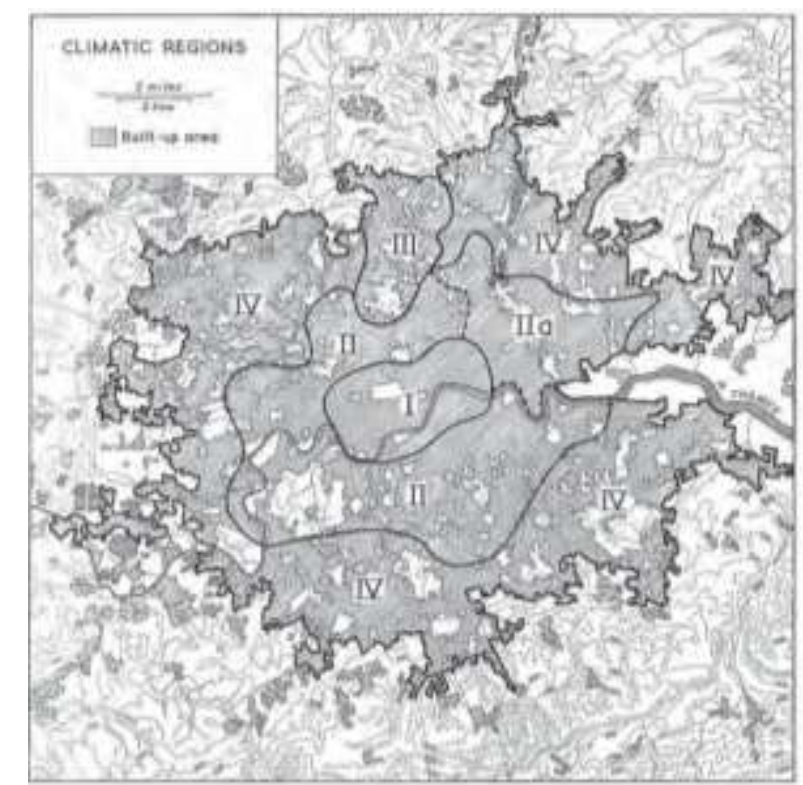

Fonte: Chandler (1965).

Aprimorando e incorporando novas informações ao sistema proposto por Ellefsen (1991), Oke (2006) sistematizou outro modelo denominado Urban Climate Zones (UCZ) ou zonas climáticas urbanas, no qual é avaliada a capacidade urbana de influenciar o clima local (figura 3). A diferença desta classificação em relação a proposta de Ellefsen (1991) foi a inserção do parâmetro "superfícies impermeáveis", que ampliou a análise em qualidade e representação, criando classes de UCZ1 (intensamente urbanizada) até UCZ7 (semi-rural).

Ao analisar estes sistemas de classificações, Stewart (2011) destaca que, apesar das muitas vantagens existentes, todos carregam limitações que dificultam a sua utilização. $O$ autor levanta especificamente quatrotópicos: (1) relevância climática, (2) a representação urbana e rural, (3) nomenclatura, e (4) origem e escopo. Nenhum dos esquemas possui equilíbrio na representação dos ambientes urbanos e rurais e todas as paisagens rurais não são, ou são pouco, elaboradas; os distintos sistemas utilizam-se de diferentes nomenclaturas, ou seja, não há padronização nos termos, o que, por sua vez, abre possibilidades de variações em cada cidade estudada e, por fim, Stewart (2011) afirma que dentre dessas classificações nenhuma possui caráter global. Mesmo sendo a classificação de Oke (2006) mais abrangente é incerto seu uso em diferentes realidades, como, por exemplo, em países subdesenvolvidos, uma vez que bairros e loteamento populares não possuem uma classe dentro da UCZ.

Em âmbito nacional são poucas as classificações das cidades voltadas ao clima encontradas na literatura. Podem ser destacados os trabalhos de Brandão e Tarifa (1995), para a cidade do Rio de Janeiro; Tarifa e Armani (2001), na cidade de São Paulo; Jardim (2007), para áreas urbanas da Bacia Hidrográfica do Rio Aricanduva em São Paulo, além de Fialho (2010), que estudou a llha do Governador-RJ. 
Figura 3 - Esquema da classificação UrbanClimate Zones (UCZ)

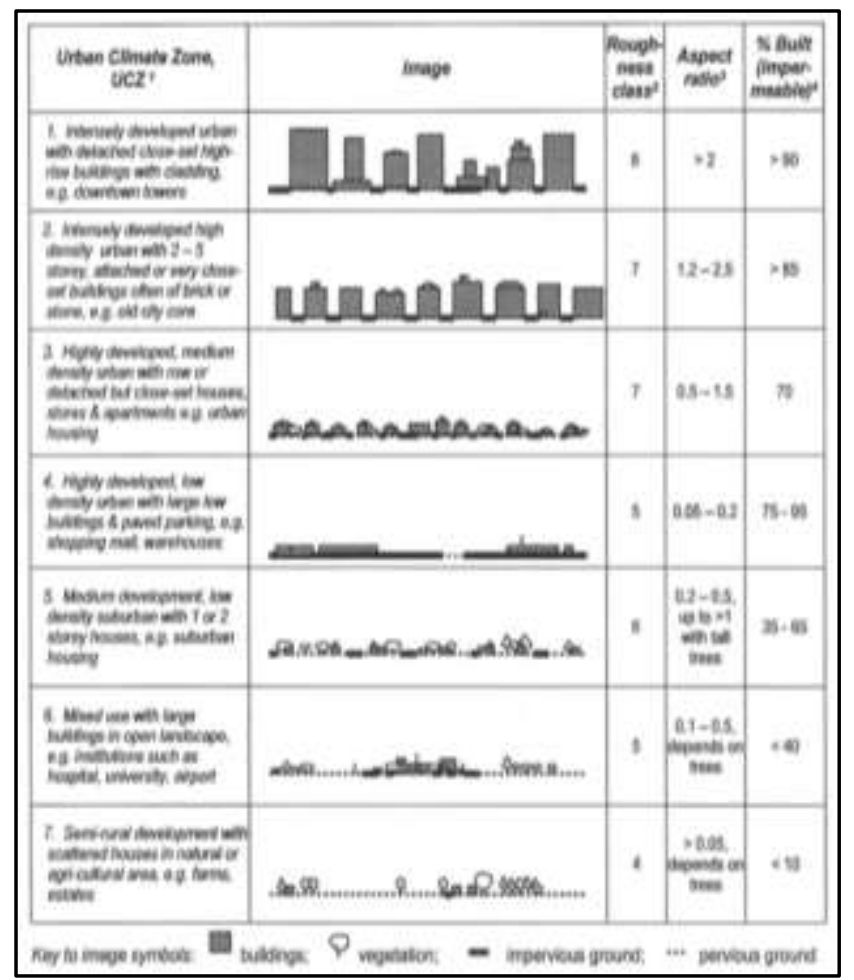

Fonte: Oke (2006).

O trabalho de Brandão e Tarifa (1995) objetivou estabelecer relações entre os aspectos geoecológicos do sítio da cidade do Rio de Janeiro e o uso da terra com as ilhas de calor detectadas. A partir de transectos móveis foram levantados dados de temperatura do ar e a partir de interpretação aerofotogramétrica foram definidos os diferentes tipos de uso do solo, os quais, foram reagrupados em 10 classes e calculados os percentuais de participação de cada tipo de uso para todas as áreas amostradas e, então, realizou-se a correlação entre o uso e a ocupação da terra e a intensidades das ilhas de calor.

Tarifa e Armani (2001), como parte do Atlas Ambiental do Município de São Paulo, elaboraram a classificação das unidades climáticas locais naturais e urbanas do município. As unidades naturais foram classificadas de acordo com os principais controles climáticos naturais da região, o Oceano Atlântico, a altitude e o relevo (enquanto forma e orientação das vertentes). No que concerne às unidades climáticas urbanas, os autores correlacionaram os controles climáticos urbanos (uso do solo, fluxo de veículos, densidade populacional, densidade das edificações, orientação e altura das edificações, áreas verdes, represas, parques e emissão de poluentes) e os elementos climáticos (temperatura da superfície, do ar, umidade, insolação, radiação solar, qualidade do ar, pluviosidade e ventilação). 
Jardim (2007) buscou identificar a influência dos fatores geográficos de superfície no padrão da temperatura e umidade relativa do ar em áreas urbanas da Bacia Hidrográfica do Rio Aricanduva em São Paulo. As cartas síntese foram elaboradas em associação ao sistema atmosférico atuante. Sendo assim, foram definidas três unidades climáticas: (I) Unidade Climática Urbana do Baixo Vale, (II) Unidade Climática Urbana do Alto Vale e (III) Unidade Climática Urbana do Fundo de Vale (unidade de transição), com as seguintes subdivisões: (la) Unidade Climática de Fundo de Vale e (Ila) Unidade Climática de Topos e Encostas.

Fialho (2010) sistematizou unidades climáticas urbanas na llha do Governador (RJ) a partir das orientações propostas apresentados em Brandão e Tarifa (1995) e Tarifa e Armani (2001). Com dados obtidos através de transectos móveis o autor elaborou uma carta de unidades topoclimáticas urbanas, a qual integrou as informações climáticas (temperatura, umidade relativa do ar e direção e velocidade do vento) aos fatores geoecológicos (posição geográfica, orientação de vertentes e altitude).

Machado e Assis (2017) apresentaram uma proposta metodológica de mapeamento de unidades topoclimáticas. Estas unidades foram delimitadas através de análise multicritérios em ambiente SIG a partir das relações existentes entre as características geoecológicas e as condições atmosféricas. As variáveis utilizadas foram radiação solar, temperatura do ar, uso e ocupação do solo, umidade relativa do ar e altimetria.

Assis e Ferreira (2018) elaboraram um estudo sobre o zoneamento térmico relacionado aos padrões de uso e ocupação da terra e a temperatura na região central de Juiz de Fora/MG. O resultado do cruzamento entre as variáveis hipsometria, albedo, edificação, vegetação, hidrografia, temperatura e sensação térmica resultaram em dois mapas com classes distintas, um referente ao conforto térmico durante o dia e outro durante a noite.

Os trabalhos até aqui apresentados partiram da aglutinação dos fatores urbanos e físicos das cidades e as informações climáticas para, então, definirem as classes específicas de cada realidade intraurbana. Deste modo, não tiveram uma abordagem completa, detalhada ou até mesmo padronizada e isso gera a dificuldade de aplicabilidade em diferentes lugares.

A fim de sanar essa lacuna e propor um sistema de classificação mais completo e abrangente com padronização de nomenclatura e definições, Stewart (2011) estabeleceu um sistema denominado de Local Climate Zones (LCZ) ou Zonas Climáticas Locais, o qual será apresentado a seguir. 


\section{AS ZONAS CLIMÁTICAS LOCAIS (LOCAL CLIMATES ZONES - LCZ)}

O principal propósito do sistema LCZ é melhorar a comunicação entre os investigadores das ilhas de calor, através de um conjunto de classes que descreva as áreas urbanas e rurais de todo o mundo.

Sendo assim, tomando o conceito mais prático de paisagem, definida como uma unidade visível, indicada por fatores naturais, sociais e culturais, Stewart e Oke (2009) criaram uma classificação climática urbana centrada nas propriedades que influenciam o campo térmico do dossel urbano, a Local Climates Zones (LCZ) ou Zonas Climáticas Locais.

Os autores distinguem as diferentes paisagens de acordo, inicialmente, com o grau de modificação da superfície, ou seja, o aspecto técnico-cultural se sobrepondo à superfície físico-natural (COLLISCHONN; MATTOS, 2011). Partindo deste princípio e baseados nas duas principais características que influenciam o clima das cidades, morfologia da superfície e cobertura do solo, Stewart e Oke (2009), levantaram quatro propriedades de superfície que são relevantes para os estudos de clima urbano: 1. altura dos elementos de rugosidade; 2. distribuição dos elementos de rugosidade; 3. cobertura da terra ao redor dos elementos de rugosidade; 4. inércia térmica dos materiais construtivos (figura 4).

Figura 4 - Propriedades relevantes encontradas na superfície segundo Stewart e Oke (2009)

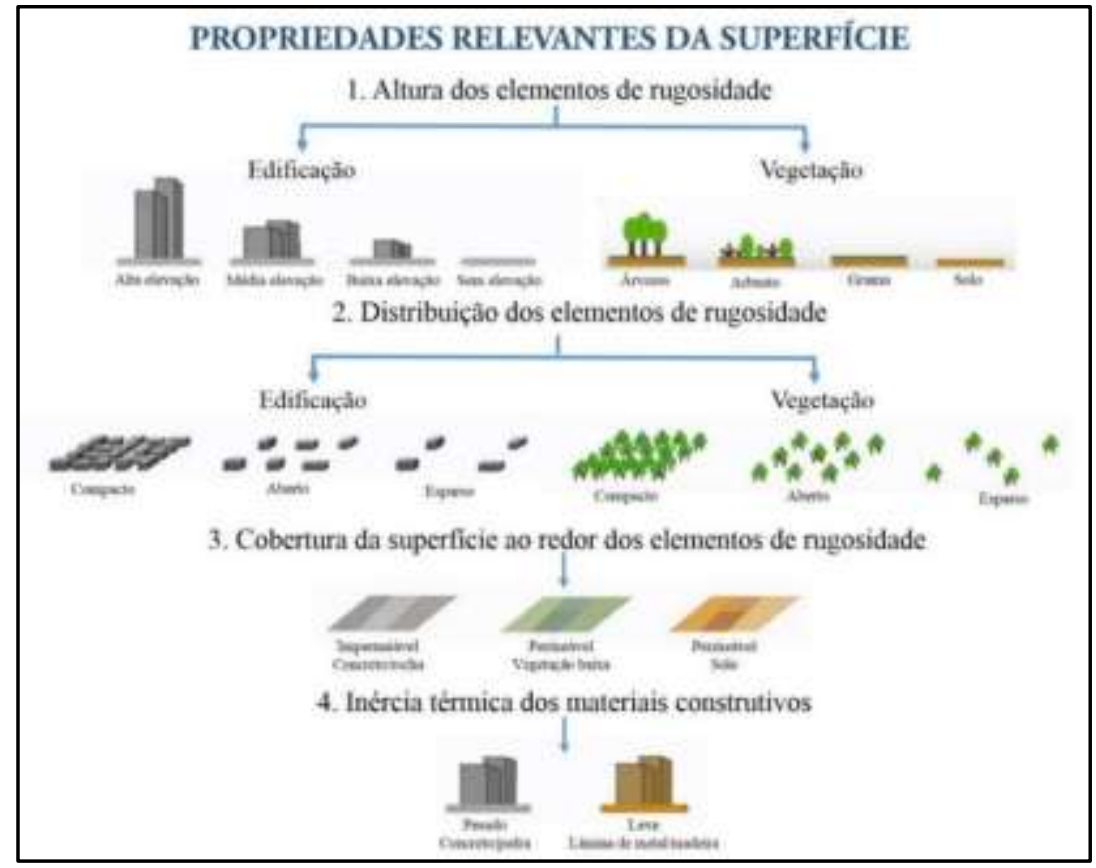

Fonte: Dorigon (2019).

Os autores elaboraram, ao todo, $17 \mathrm{LCZs}$, as quais representam a diversidade das paisagens urbanas. Interessante destacar que essa denominação foi utilizada pelo autor, 
pois elas são (L) locais na escala, (C) climáticas na distinção das propriedades e $(Z)$ zonais na representação espacial.

Durante cinco anos, de 2005 a 2010, o autor aperfeiçoou o sistema de classificação através da aplicação em Uppsala (Suécia), Nagano (Japão) e Vancouver (Canadá), onde temperaturas do ar foram observadas e simuladas através de modelos atmosféricos de mesoescala. O resultado, apresentado na tese de doutorado de Stewart (2011), sistematizou 17 LCZs, das quais 10 são relacionadas aos tipos de construções $(1-10)$ e 7 referentes aos tipos de cobertura da terra $(A-G)$. Além dessas categorias, o autor enumerou uma classe para o uso industrial e 4 subdivisões que podem ser usadas para caracterizar propriedades sazonais de cobertura da terra (figura 5).

Figura 5 - As zonas climáticas locais (LCZ)

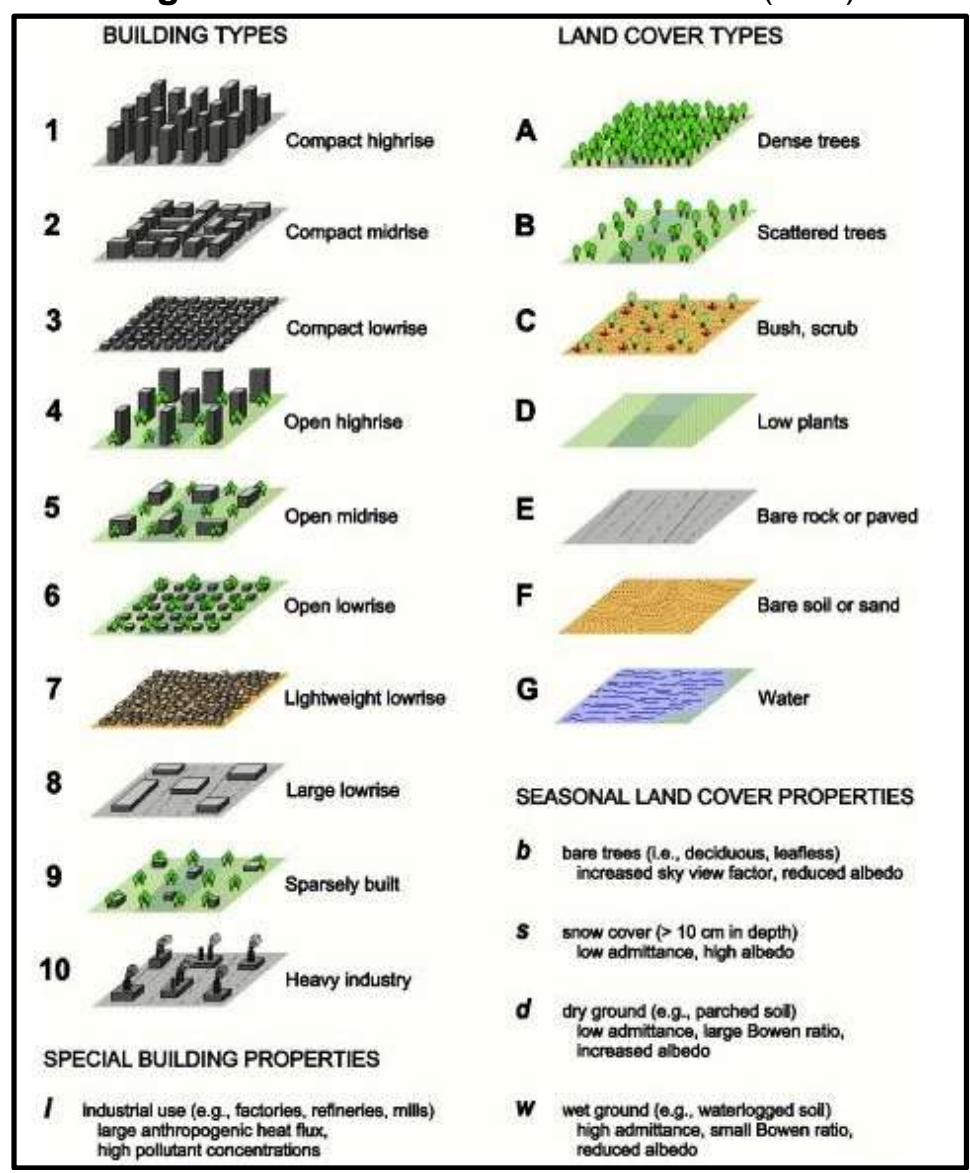

Fonte: Stewart (2011).

Os tipos 1 a 10, que correspondem às Zonas Climáticas Urbanas de Oke (2006), são ordenados de formas densas/altas para formas abertas/baixas. Os tipos de cobertura da terra A - G são naturais com pouca cobertura (ou nenhuma) de estradas ou edifícios e sem emissões antropogênicas de calor. Estes são ordenados de acordo com a aerodinâmica, ou seja, de rugosos a lisos e podem ser subclassificados em propriedades sazonais (isto é, vegetação com/sem folhas, solo seco/úmido, solo coberto de neve) (STEWART, 2011). 
Segundo o autor, a uniformidade retratada em cada LCZ é improvável de ser encontrada na realidade, exceto em locais planejados ou monitorados. No entanto, os 17 padrões de LCZs devem ser familiares para os pesquisadores das ilhas de calor na maioria das cidades, e devem ser adaptáveis ao caráter local da maioria dos locais. Para garantir sua aplicabilidade em outras realidades, o sistema LCZ foi construído com partes componentes que são combinadas em subclasses (figura 6). De acordo com ele,

Se necessário, combine classes de LCZ o mostre como o local se difere de seu equivalente mais próximo no conjunto padrão. Considere os tipos de construção, propriedades especiais de construção, tipos de cobertura da terra e propriedades de cobertura sazonal (STEWART, 2011, p. 254, tradução nossa).

Figura 6 - Possibilidade de subclassificação das LCZs

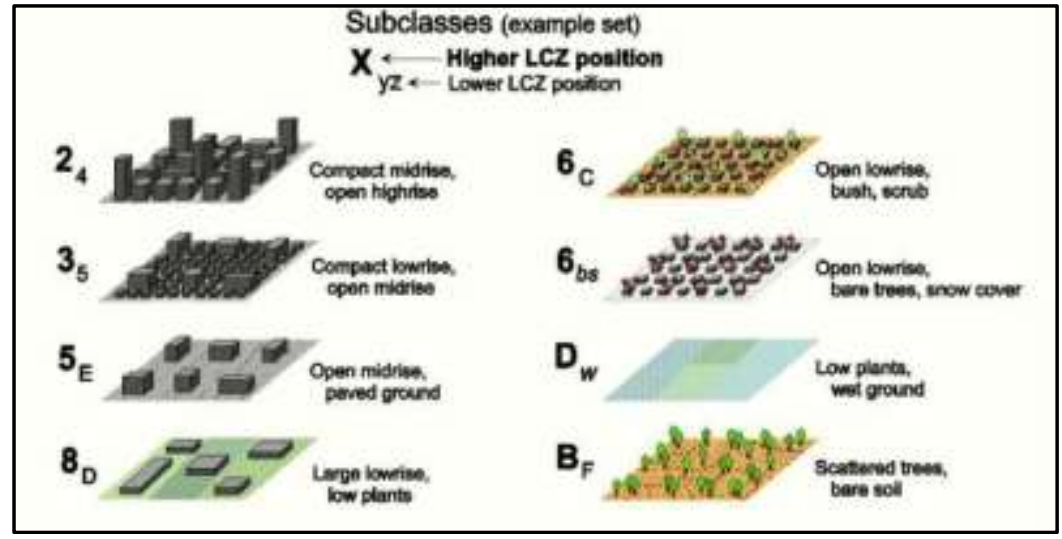

Fonte: Stewart, 2011.

Após a classificação feita, Stewart (2011) mostrou que a elaboração de folhas sínteses é uma maneira eficaz de apresentar os resultados, além de promover a comunicação padronizada entre os pesquisadores do tema (figura 7). Estas folhas são utilizadas para representação visual de todas as informações contidas nas diferentes classes, traçando, assim, o perfil individual de cada uma e devem conter as seguintes informações: identificação (nome da classe e o código), definição (características morfológicas da superfície, cobertura da terra e fluxo antropogênico), função (comercial, residencial e agrícola); localização (cidade, campo, centro e periurbano), ilustração (desenhos e fotografias) e propriedades da superfície (altura da rugosidade superficial, fração de superfície impermeável e características térmicas). Na sequência apresenta-se a folha síntese elaborada por Stewart (2011) e disponibilizada em sua tese para ser utilizada como modelo nos trabalhos a serem desenvolvidos (figura 7).

Desde 2011 e, principalmente, após a publicação do artigo Local Climate Zones for Urban Temperature Studies (STEWART; OKE, 2012), muitas pesquisas vêm sendo publicadas e desenvolvidas utilizando a proposta, sendo que atualmente existe uma 
ferramenta online a fim de reunir em uma plataforma os estudos elaborados baseados nas LCZs. De acordo com o próprio site (www.wudapt.org/) o projeto WUDAPT (The World Urban Database and Access Portal Tools) busca alimentar um banco de dados baseados nos trabalhos da comunidade acadêmica interessada, além de facilitar a elaboração dos censos das cidades através de workshops.

Figura 7 - Exemplo de folha síntese

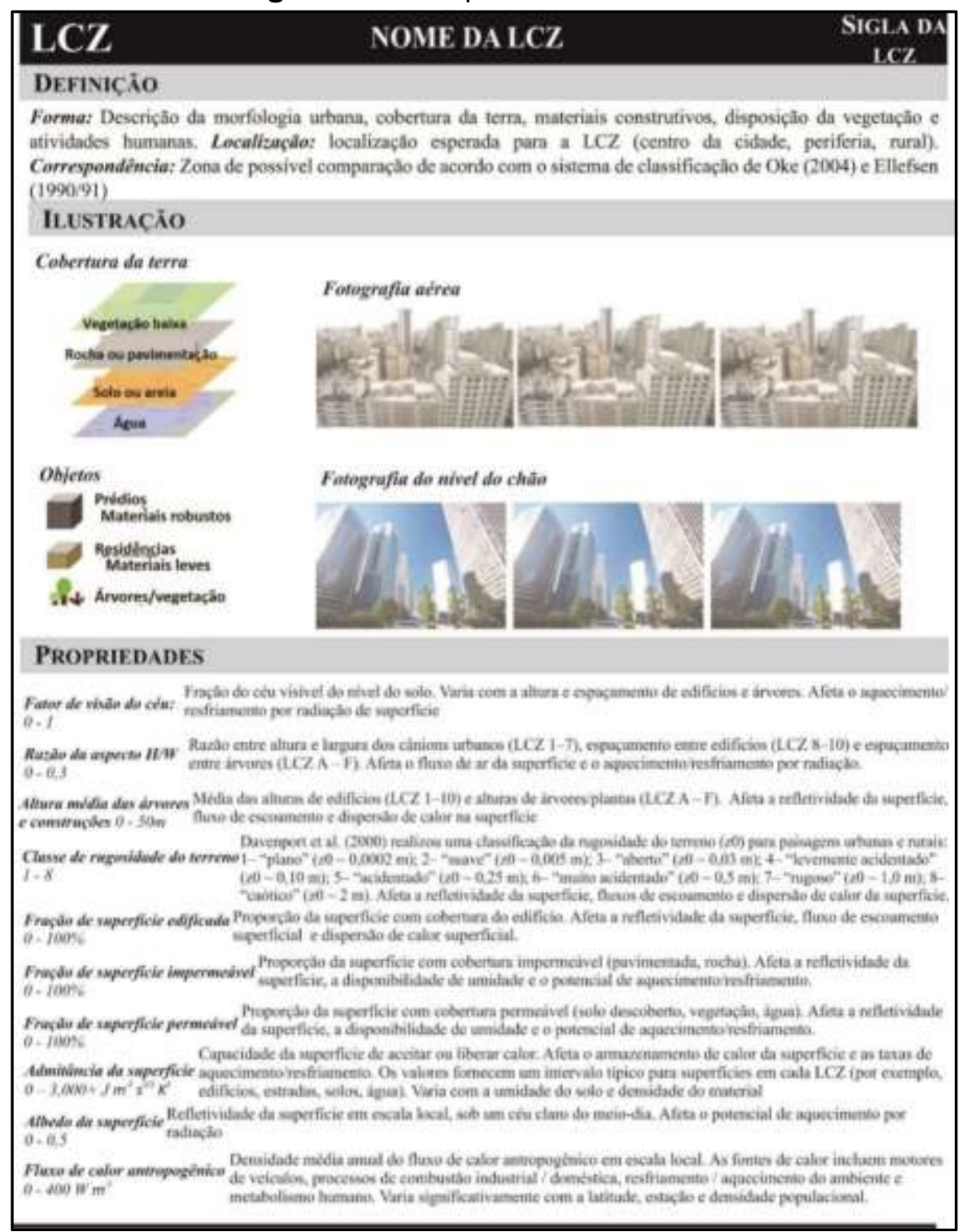

Fonte: Stewart (2011, tradução nossa).

Partindo dos resultados de pesquisas que utilizaram de sua metodologia e, principalmente, como consequência do workshop da WUDAPT realizado na Universidade de Hong Kong em 2015, Stewart (2018) apresentou na 10ª Conferência Internacional de Clima Urbano (10th International Conference on Urban Climate), realizada em agosto de 2018, na 
Universidade de Nova lorque, um paper no qual o autor elaborou um guia para auxiliar na identificação das LCZs em campo.

Dois são os principais pontos abordados neste novo guia. O primeiro é a estruturação do pensamento, o autor indica que é necessário responder questões sobre uma ou mais características da área urbana (ou rural) a ser classificada. As perguntas são emparelhadas com exercícios cognitivos que levam a observação e interação com a área a ser classificada, e então, pode-se gerar julgamentos razoáveis sobre a classe LCZ à qual a área pertence (figura 8).

Figura 8 - Exemplo de perguntas e exercícios cognitivos do novo guia da LCZ

\begin{tabular}{|c|c|}
\hline \multicolumn{2}{|c|}{ 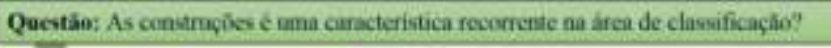 } \\
\hline 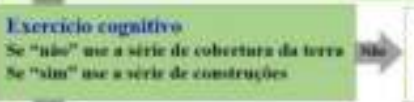 & $\begin{array}{l}\text { Questâa: As árvores de uma caracteristica } \\
\text { recoerente na drea de classificaçăo? } \\
\text { Exercicie cognitivo }\end{array}$ \\
\hline \multicolumn{2}{|c|}{ ver } \\
\hline \multicolumn{2}{|c|}{ 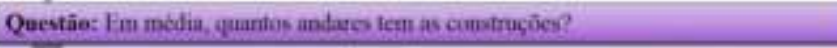 } \\
\hline $\begin{array}{l}\text { Exercicio cognitivas } \\
\text { Se }>1 \text {, use Tpes Alta'mésfies } \\
\text { Se S uac Tipms buiun }\end{array}$ & $\begin{array}{l}\text { Questáo: Em média, qual a distlincia entre } \\
\text { os edificios mais alion? } \\
\text { Exercicie cognitivo }\end{array}$ \\
\hline \multicolumn{2}{|l|}{+7} \\
\hline \multicolumn{2}{|c|}{ Quetäa: O espaçamenio entre as construçèes baixas é grande" } \\
\hline $\begin{array}{l}\text { Exercicio cognitivo } \\
\text { Se "nàa", ase Tipas Abertos } \\
\text { Se "vim", use LCZ8 }\end{array}$ & $\begin{array}{l}\text { Questîo: Em midia, qual a distaincia entre } \\
\text { os edificios mais baixo? } \\
\text { Exercicie cognitivo }\end{array}$ \\
\hline
\end{tabular}

Fonte: Stewart (2018, tradução nossa).

O segundo ponto, extremamente importante, é a inserção do relevo nas LCZs. Este novo guia apresenta o esquema anterior das LCZs e acrescenta características físicas: elevação, forma do terreno e inclinação (figura 9). De acordo com o autor, "Com essa elaboração, pode-se usar o esquema para separar e explicar as diferenças locais nas temperaturas do ar medidas que são devidas a efeitos urbanos e não-urbanos" (STEWART, 2018, p. 5, tradução nossa).

Stewart (2011), em suas conclusões, levanta algumas questões e necessidades para futuras pesquisas e a empregabilidade dessa classificação. Algumas delas se referem aos testes e aplicações em cidades tropicais e desérticas e também em cidades do mundo em desenvolvimento. 
Figura 9 - Classes dos relevos locais junto as LCZs

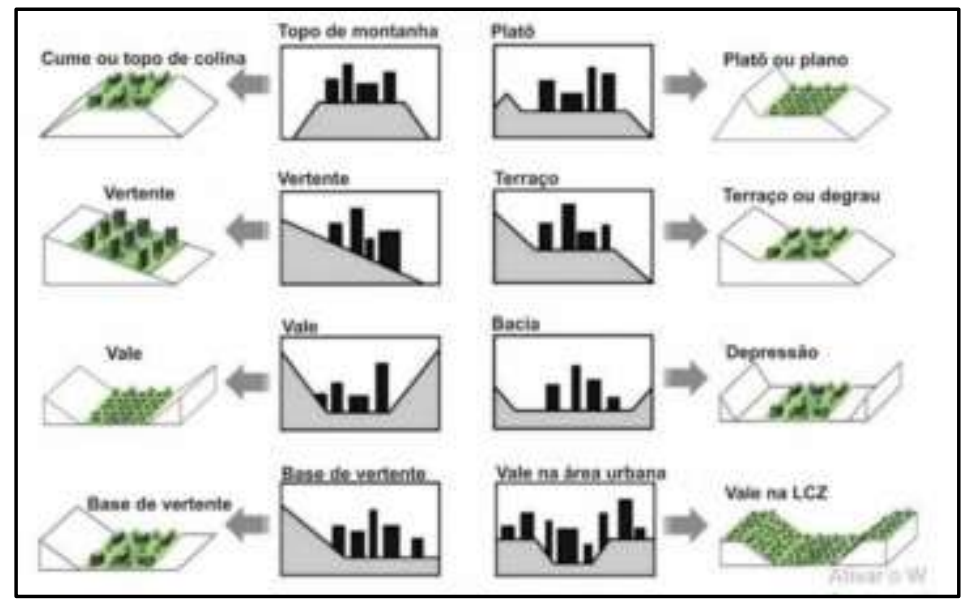

Fonte: Stewart (2018, tradução nossa).

\section{EXEMPLOS DE APLICAÇÕES DAS ZONAS CLIMÁTICAS LOCAIS (LOCAL CLIMATES ZONES - LCZ)}

A metodologia desenvolvida Stewart e Oke (2012) vem sendo aplicada em diversos estudos nacionais e internacionais. Collischonn e Mattos (2011) publicaram o artigo "Classificação de ambientes termicamente homogêneos para estudos de clima na camada do dossel urbano - metodologia e aplicação à cidade de Pelotas/RS" (figura 10). A proposta do trabalho foi realizar uma revisão de literatura sobre as classificações de unidades climáticas urbanas, em especial sobre a proposta de Stewart e Oke (2009) - Zonas climáticas locais (Local Climate Zones - LCZ) - e, então, foram feitas adaptações para se realizar a classificação para o perímetro urbano de Pelotas. A partir de imagens Ikonos II PSM de alta resolução foi elaborado um mapa com as Potenciais Unidades Climáticas (baseadas nas Local Climate Zones) e pode-se tecer considerações a respeito das ilhas de calor ali detectadas.

Cardoso (2015) e Anjos (2017) elaboraram seus trabalhos de conclusão de mestrado e doutorado, respectivamente, a partir das LCZs. Cardoso (2015) apresentou um mapa de Potenciais Unidades Climáticas de Presidente Prudente que foi elaborado segundo a proposta de Stewart e Oke (2012) e teceu considerações sobre as ilhas de calor na cidade (figura 11).

Anjos (2017) defendeu sua tese de doutoramento pela Universidade de Lisboa e elaborou propostas de planejamento climático para a cidade de Aracajú, utilizando as LCZs como base para a caracterização de uso e ocupação da terra (figura 12). Vale destacar que este autor faz uma avaliação sobre essa metodologia e explicita que as LCZs são baseadas no conhecimento simplificado e generalista sobre o uso da terra e estrutura urbana e acabam por excluir a topografia e a climatologia local. No entanto, elas se mostram 
vantajosas pelo fato de que o sistema é universal, o que permite a comparação entre locais diferentes do mundo e fornece informações ou metadados úteis (propriedades térmicas, radiativa, geométrica, metabólica e de cobertura da superfície).

Figura 10 - Potenciais unidades climáticas urbanas de Pelotas

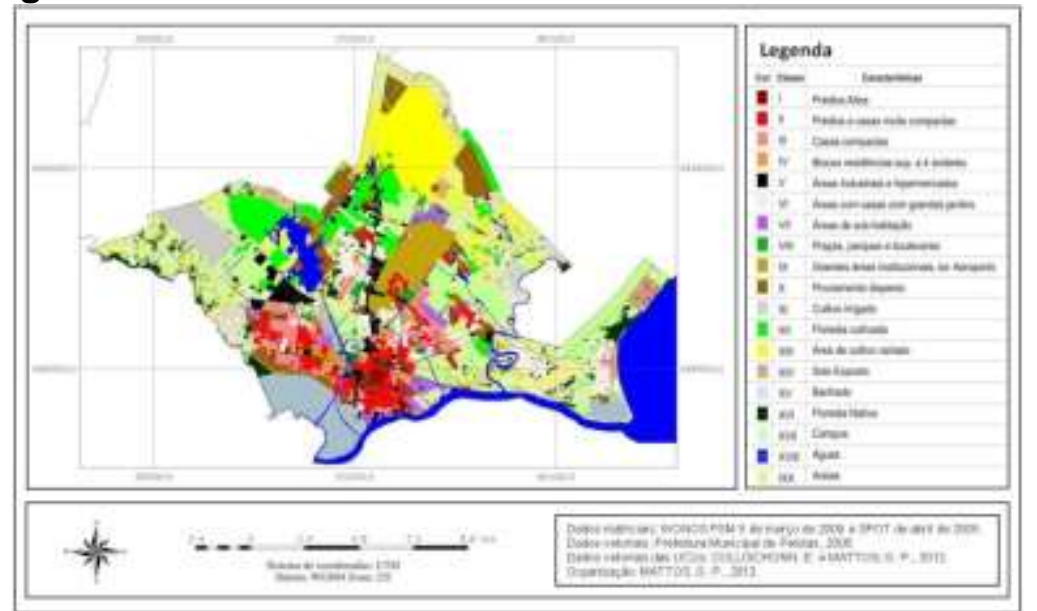

Fonte: Collischonn e Mattos (2011).

Figura 11 - Mapa das Potenciais Unidades Climáticas em Presidente Prudente

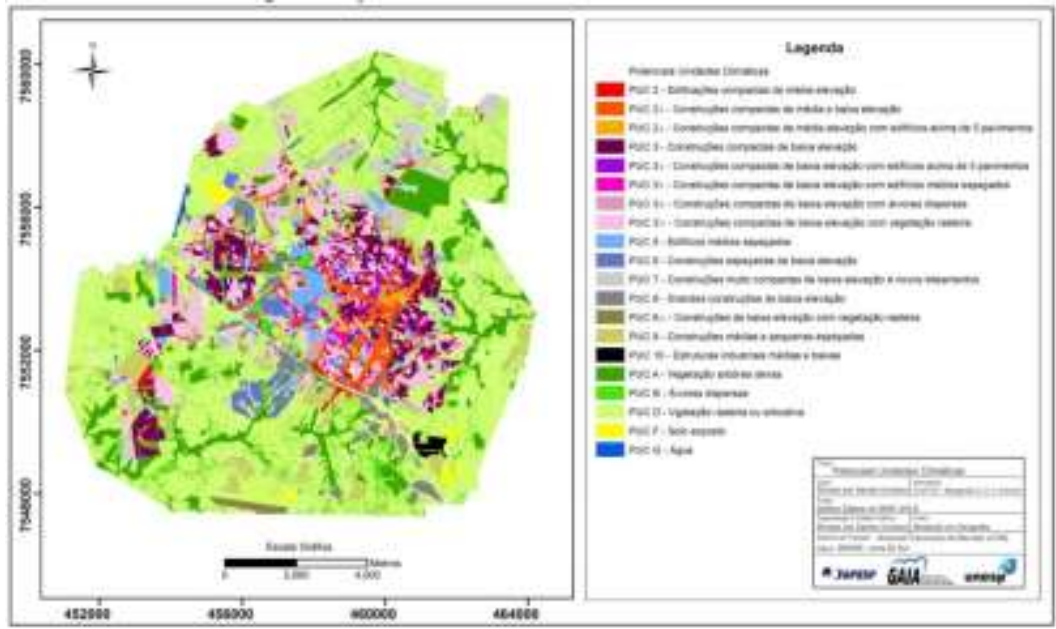

Fonte: Cardoso (2015).

Monteiro (2018) desenvolveu uma pesquisa com o objetivo de investigar a distribuição espacial da temperatura do ar e superficial e dos padrões de uso e ocupação da terra em uma área do município de Campinas. Para realizar esta correlação entre temperatura e uso e ocupação da terra foi utilizada a classificação das LCZs. Interessante destacar que, neste trabalho, o enfoque da autora foi discutir as características de cada uma das LCZs em relação aos parâmetros climáticos medidos (temperatura e umidade), ao contrário da maioria das pesquisas desenvolvidas que buscaram relacionar os elementos climáticos às características de cada LCZs. 
Figura 12 - Mapa das Local Climate Zones em Aracajú

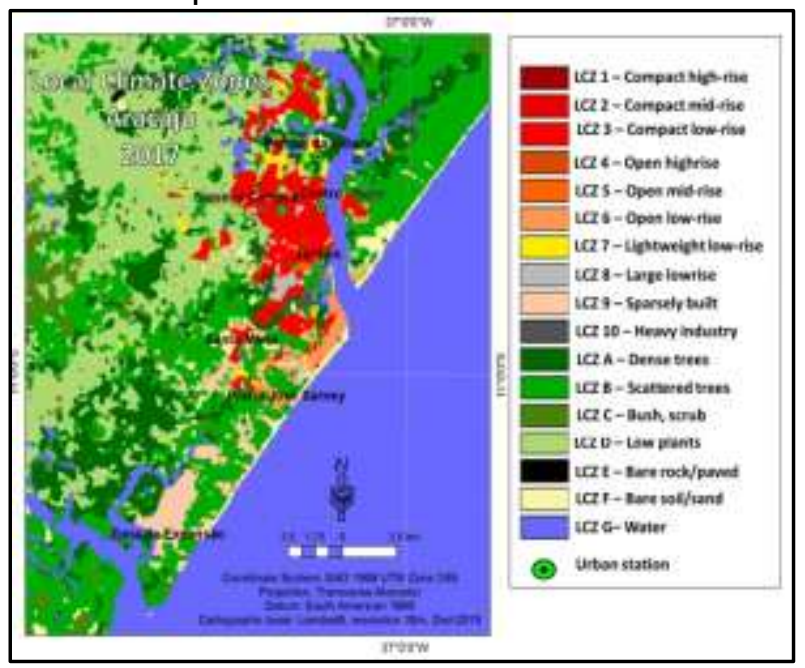

Fonte: Anjos (2017).

Dorigon (2019), em sua tese de doutorado, teve como uma de suas hipóteses que a utilização da metodologia das Zonas Climáticas Locais (LCZs) facilita a comparação dos resultados obtidos em Jundiaí com outras áreas urbanas, uma vez que esta proporciona a uniformização e padronização de terminologias referentes aos usos e ocupações da terra. Sendo assim, a autora utilizou esta metodologia para identificar os usos e as ocupações da terra no local de estudo. Tecendo considerações sobre essa metodologia, a autora afirma que as LCZs conseguem facilitar o intercâmbio dos resultados obtidos, uma vez que padroniza a nomenclatura, no entanto, as suas delimitações podem ser bastante subjetivas.

$\mathrm{Na}$ literatura internacional a utilização das LCZs é bastante difundida em trabalhos que buscam compreender as ilhas de calor de urbanas, seja através da correlação entre as características de uso e ocupação da terra e as temperaturas locais (do ar ou superficiais) (KOTHARKAR; BAGADE, 2018; MADUREIRA et al., 2017; MIDDEL et al., 2014; MONTEIRO et al., 2018; NG, 2015; ZHONGLI; HANQIU, 2016), seja pela elaboração de modelos para a compreensão do fenômeno (FOISSARD, 2015), além do desenvolvimento de novas tecnologias e a utilização de imagens de satélite para o aprimoramento da identificação das LCZs (BECHTEL, 2011; BECHTEL et al., 2015; XU, 2017; WANG et al., 2018).

Estes trabalhos abordam diferentes áreas urbanas em contextos climáticos variados ao redor do mundo, como, por exemplo, o estudo de Stevan et al. (2013), na cidade de Novi Sad/Servia; Perera, Emmanuel e Mahanama (2012) em Colombo/Sri Lanka; Ng (2015) em Singapura; muitos elaborados para cidades europeias, por exemplo: Geletič, Lehnert e Dobrovolný (2016); Houeta e Pigeon (2011); Lehnert, Geletič e Husák (2016); Madureira et al. (2017), além dos estudos realizados na América do Norte (MIDDEL et al., 2014; STEWART; OKE, 2009) e na Ásia, especialmente na China (CAl et al., 2018; ZHONGLI; 
HANQIU, 2016). Na sequência estão apresentados alguns dos mapas de LCZ (quadro 1) elaborados nos artigos citados, sendo estes: Cai et al. (2018); Geletič, Lehnert e Dobrovolný (2016); Madureira et al. (2018); Middel et al. (2014); Monteiro et al. (2018); Zhongli e Hanqiu (2016).

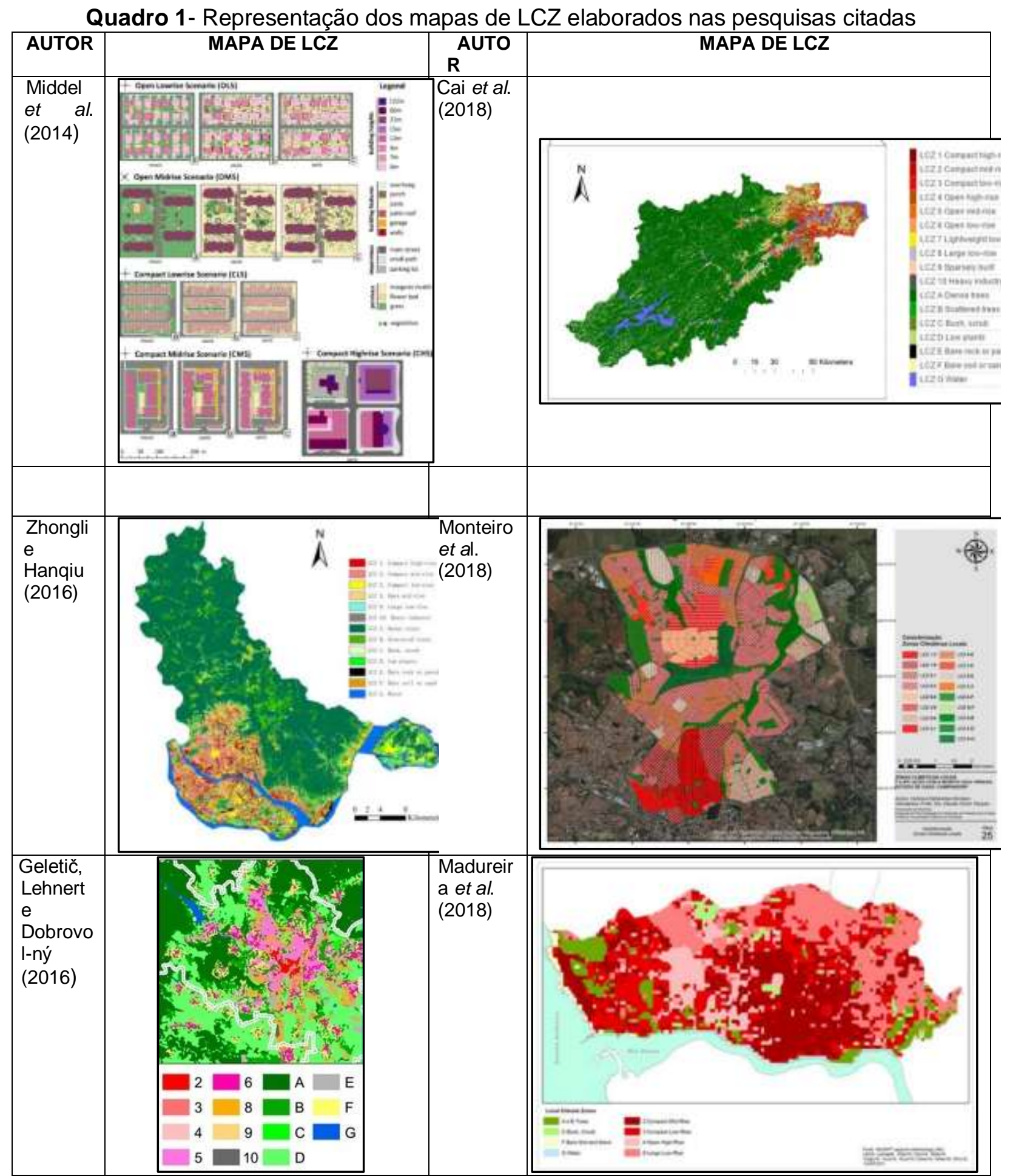

Fonte: Cai et al. (2018); Geletič, Lehnert e Dobrovolný (2016); Madureira et al. (2018); Middel et al. (2014); Monteiro et al. (2018); Zhongli e Hanqiu (2016). 


\section{CONSIDERAÇÕES FINAIS}

Ainda que, atualmente, os estudos sobre as ilhas de calor sejam difundidos e aprofundados, os procedimentos de investigação e comunicação podem ser aperfeiçoados a fim de facilitar a documentação consistente dos dados.

Stewart (2011) confirmou a limitação do uso da classificação tradicional "urbano-rural", visto que esses dois ambientes são distintos em cada localização do planeta. Para ultrapassar essa limitação, o autor propôs o sistema de classificação da paisagem denominado "Local Climate Zones" (LZC), ou Zonas Climáticas Locais, através do qual a paisagem é dividida de acordo com as propriedades da morfologia da superfície e cobertura da terra. Essa proposta se constitui um guia prático para a classificação dos locais de medição de temperatura para as investigações das ilhas de calor.

Sendo assim, a utilização da metodologia das LCZs possibilita melhor descrição das áreas investigadas, dada a diversidade de características da superfície apresentada no sistema, além de promover a divulgação e a comunicação mais eficiente das análises, proporcionando comparações entre os resultados de pesquisas desenvolvidas nas diferentes localidades e auxiliando na elaboração de medidas mitigadoras e no planejamento urbano.

Contudo, apesar de a proposta buscar englobar todas as possibilidades de ocupações urbanas, as suas aplicações não necessariamente contemplaram todos os ambientes, assim como detectado por Dorigon (2019) e por isso, a delimitação das LCZs pode ser bastante subjetiva.

\section{REFERÊNCIAS}

ANJOS, Max Wendell Batista. Orientações climáticas para o planejamento urbano numa cidade costeira do nordeste do Brasil: Aracaju-SE. Tese (Doutorado em Geografia) Universidade Nova de Lisboa, Lisboa, 2017.

AMORIM, Margarete Cristiane Costa Trindade. O clima urbano de Presidente

Prudente/SP. Tese (Doutorado em Geografia) - Faculdade de Filosofia, Letras e Ciências Humanas, Universidade de São Paulo, São Paulo, 2000.

AMORIM, Margarete Cristiane Costa Trindade. Climatologia e gestão do espaço urbano. Mercator, Fortaleza, v. 9, n. 10, p. 71-90, 2010. Disponível em: https://www.redalyc.org/pdf/2736/273620609006.pdf. Acesso em: 22 jun. 2016.

AMORIM, Margarete Cristiane Costa Trindade; DUBREUIL, Vincent; QUENOL, Hervé; SANT'ANNA, João Lima. Características das ilhas de calor em cidades de porte médio: exemplos de Presidente Prudente (Brasil) e Rennes (França). Confins [Online], [s. I.], n. 7, p. 1-16, 2009. Disponível em: <http://confins.revues.org/index6070.html>. Acesso em: 06 set. 2015. 
AMORIM, Margarete Cristiane Costa Trindade.Teoria e método para o estudo das ilhas de calor em cidades tropicais de pequeno e médio porte. Tese (Livre-Docência) Universidade Estadual Paulista, Presidente Prudente, 2017.

ARNFIELD, John. Two decades of urban climate research: a review of turbulence, exchanges of energy and water, and the urban heat island. International Journal of Climatology, Chichester, v. 23, n. 1, p. 1-26, 2003.

ASSIS, Débora Couto; FERREIRA, Cassia Castro Martins. Zoneamento térmico e suas correlações associadas aos padrões de uso e ocupação da terra da região urbana, Centro em Juiz de Fora - MG. Revista Brasileira de Climatologia, Curitiba, v. 22, p. 554-573, 2018.

AUER Jr, August H. Correlation of land use and cover with meteorological anomalies. Journal Applied Climatology, [s. I.], v. 17, p. 636-643, May 1978. Disponível em: https://journals.ametsoc.org/view/journals/apme/17/5/1520-

0450_1978_017_0636_coluac_2_0_co_2.xml. Acesso em: 06 set. 2015.

BECHTEL, Benjamin. Multitemporal Landsat data for urban heat island assessment and classification of local climate zones. In: URBAN REMOTE SENSING EVENT, 2011, Munich. Anais [...]. Munich: IEEE, 2011. p. 129-132.

BECHTEL, Benjamin et al. Mapping local climate zones for a worldwide database of the form and function of cities. ISPRS International Journal of Geo-Information, [s. I.], v. 4, n. 1, p. 199-219, 2015.

BRANDÃO, Ana Maria de Paiva Macedo; TARIFA, José Roberto. O campo térmico na cidade do Rio de Janeiro. In: SIMPÓSIO BRASILEIRO DE GEOGRAFIA FíSICA APLICADA, 6., 1995, Goiânia. Anais [...]. Goiânia: UFG, v. 2, p. 45-49, 1995.

$\mathrm{CAI}$, Meng et al. Investigating the relationship between local climate zone and land surface temperature using an improved WUDAPT methodology - A case study of Yangtze River Delta, China. Urban Climate, [ S. I.], v. 24, p. 485-502, june 2018.

CARDOSO, Renata Santos. Classificação de potenciais unidades climáticas em Presidente Prudente-SP. 2015. Dissertação (Mestrado em Geografia) - Universidade Estadual Paulista Júlio de Mesquita Filho, Presidente Prudente, 2015.

CHANDLER, Tony John. The climate of London. London: Hutchinson, 1965. Disponível em: http://urban-climate.org/documents/TonyChandler_TheClimateOfLondon.pdf. Acesso em: 23 out. 2014.

CHATEL, Cathy; SPOSITO, Maria Encarnação Beltrão. Forma e dispersão urbanas no Brasil: fatos e hipóteses. Primeiros resultados do banco de dados BRASIPOLIS. Cidades, Presidente Prudente, v. 12, n. 21, p. 108-152, 2015.

COLLISCHONN, Erika; MATTOS, Gil Passos. Classificação de ambientes termicamente homogêneos para estudos de clima na camada do dossel urbano - metodologia e aplicação à cidade de Pelotas/RS. Revista Brasileira de Climatologia, Curitiba, v. 9, p. 56-67, 2011.

DORIGON, Larissa Piffer. As ilhas de calor urbanas em Jundiaí/SP. Tese (Doutorado em Geografia) - Universidade Estadual Paulista Júlio de Mesquita Filho, Presidente Prudente, 2019.

ELLEFSEN, Richardi. Mapping and measuring buildings in the urban canopy boundary layer in ten US cities. Energy and Buildings, Lausanne, v. 16, n. 3/4, p. 1025-1049, 1991.

FIALHO, Edson Soares. Unidades climáticas urbanas: o caso da llha do Governador-RJ.

Revista de Ciências Humanas, Viçosa, v. 10, n 1, p. 26-46, 2010. Disponível em: http://www.cch.ufv.br/revista/pdfs/artigo2vol10-1.pdf. Acesso em: 22 jun. 2016. 
FOISSARD, Xavier. L'îlot de chaleururbain et le changementclimatique: application à I'agglomérationrennaise. 2015. These (Docteur) - Université de Rennes II, Rennes, 2015.

GELETIČ, Jan; LEHNERT, Michael; DOBROVOLNÝ, Petr. Land Surface Temperature Differences within Local Climate Zones, Based on Two Central European Cities. Remote Sensing, Lower Hut, v. 8, n. 10, p. 1-18, 2016.

GRIMMOND, Sue. Urbanization and global environmental change: local effects of urban warming. Geographical Journal, London, v. 173, n. 1, p. 83-88, mar. 2007.

HOUET, Thomas; PIGEON, Grégoire. Mapping urban climate zones and quantifying climate behaviours - An application on Toulouse urban area (France). Environmental Pollution, Barking, v. 159, n. 8/9, p. 2180-2192, aug./sept. 2011.

IBGE. Censo demográfico: 2000: características gerais da população: resultados da amostra. Brasília: IBGE, 2000. Disponível em: https://biblioteca.ibge.gov.br/index.php/biblioteca-catalogo?view=detalhes\&id=783. Acesso em: 02 maio 2018.

IBGE. Censo Demográfico: 2010: características da população e dos domicílios: resultados do universo. Brasília: IBGE, 2010. Disponível em:

https://biblioteca.ibge.gov.br/index.php/biblioteca-catalogo?view=detalhes\&id=793. Acesso em: 02 maio 2018.

JARDIM, Carlos Henrique. Proposta de síntese climática a partir do comportamento térmico e higrométrico do ar em áreas urbanas. 2007. Dissertação (Doutorado em Geografia) - Instituto de Geociências, Unicamp, Campinas, 2007.

KOTHARKAR, Rajashree; BAGADE, Anurag. Evaluating urban heat island in the critical local climate zones of an Indian city. Landscape and Urban Planning, Amsterdam, v. 169, p. 92104, jan. 2018.

LIN, Xue-Chun.; YU, Shu-Qiu. Interdecadal changes of temperature in the Beijing region and its heat island effect. Chinese Journal of Geophysics, [S. I.], v. 48, n. 1, p. 47-54, jan. 2005.

MACHADO, Lilian Aline; ASSIS, Wellington Lopes. Proposta metodológica de mapeamento de unidades topoclimáticas. Revista Brasileira de Climatologia, Curitiba, v. 21, p. 186-204, 2017.

MADUREIRA, Helena et al. Identificação de 'Local Climate Zones' (LCZ's) no concelho do Porto. In: CONGRESSO DA GEOGRAFIA PORTUGUESA, 11., 2017. Porto. Anais [...], Porto: Universidade do Porto, 2017. p. 453-456.

MENDONÇA, Francisco; DUBREUIL, Vicenti. Termografia de superfície e temperatura do ar na RMC (Região Metropolitana de Curitiba - PR). RA'E GA - O espaço geográfico em análise, Curitiba, n. 9, p. 25-35, dez. 2005.

MIDDEL, Ariane et al. Impact of urban form and design on mid-afternoon microclimate in Phoenix Local Climate Zones. Landscape and Urban Planning, Amsterdam, v. 122, p. 1628, Feb. 2014.

MONTEIRO, Ana et al. (coord.). O(s) clima(s) de Gaia. Vila Nova de Gaia: Câmara Municipal, 2018. Disponível em: https://repositorioaberto.up.pt/bitstream/10216/117285/2/301089.pdf. Acesso em: 02 maio 2018.

MONTEIRO, Carlos Augusto de Figueiredo. Teoria e clima urbano. São Paulo: IGEOG/USP, 1976.

MONTEIRO, Verônica Stefanichen. Zonas climáticas locais e a relação com a morfologia urbana. Estudo de caso: Campinas/SP. 2018. Dissertação (Mestrado em 
Sistemas de Infraestrutura Urbana) - Pontifícia Universidade Católica de Campina, Campinas, 2018.

NG, Yvonne. A Study of Urban Heat Island using "Local Climate Zones" - The Case of Singapore. International Journal of Environment and Climate Change, [S. I.], v. 5, n. 2, p. 116-133, 2015.

OKE, Tim Richard. Initial guidance to obtain representative meteorological observations at urban sites. Geneva: World Meteorological Organization, 2006. Disponível em: http://www.wmo.int/pages/prog/www/IMOP/publications/IOM-81/-UrbanMetObs.pdf. Acesso em: 12 ago. 2016.

ONU-HABITAT. Relatório Estado das Cidades da América Latina e Caribe. Produzido pelo Programa das Nações Unidas para os Assentamentos Humanos. Disponível em: $<$ https://www.mobilize.org.br/midias/pesquisas/estado-das-cidades-da-america-latina-ecaribe-2012.pdf>. Acesso em: 14 set. 2016.

PERERA, Narein; EMMANUEL, Rohinton; MAHANAMA, P. K. S. Mapping "Local Climate Zones" and relative warming effects in Colombo, Sri Lanka. In: INTERNATIONAL CONFERENCE ON URBAN CLIMATE, 8., 2012. Dublin. Anais [...]. Dublin: [s. n.], p.1-6, 2012.

REN, Guoya; CHU, Ziying; CHEN, Yaning. Implications of temporal change in urban heat island intensity observed at Beijing and Wuhan stations. Geophysical Research Letters, Washington, v. 34, n. 5, p. 1-5, Mar. 2007. Disponível em:

https://agupubs.onlinelibrary.wiley.com/doi/epdf/10.1029/2006GL027927. Acesso em: 14 set. 2016.

REN, Guoya; ZHOU, Yaqing. Urbanization effect on trends of extreme temperature indices of national stations over mainland China, 1961 e 2008. International Journal of Climatology, Chichester, v. 27, p. 2340-2360, mar. 2014.

SANTOS, Milton. Técnica, espaço, tempo: globalização e meio técnico científico informacional. São Paulo: Hucitec, 1993.

STEVAN, Savic et al. Classifying Urban Meteorological Stations Sites by "Local Climate Zones": Preliminary Results for the City of Novi Sad (Serbia), Geographica Pannonica, [S. I.], v. 17, n. 3, p. 60-68, sept. 2013.

STEWART, lan Douglas; OKE, Tim Richard. Newly developed "thermal climate zones" for defining and measuring urban heat island magnitude in the canopy layer. In: SYMPOSIUM \& EIGHTH SYMPOSIUM ON URBAN ENVIRONMENT, 2009, Vancouver. Anais [...]. Vancouver: University of British Columbia, 2009. p. 1-6.

STEWART, Ian Douglas; OKE, Tim Richard. Local Climate Zones for Urban Temperature Studies. Bulletin of the American Meteorological Society, Boston, v. 93, n.12, p.1879-1900, Dec. 2012. Disponível em:

https://journals.ametsoc.org/doi/pdf/10.1175/BAMS-D-11-00019.1. Acesso em: 12 ago. 2015.

STEWART, lan Douglas. Redefining the urban heat Island. 2011. Thesis (Doctor of Philosophy) - The Faculty of Graduate Studies, The University of British Columbia, Vancouver, 2011.

STEWART, lan Douglas. Developing a field guide to identify 'local climate zones' in cities. In: INTERNATIONAL CONFERENCE ON URBAN CLIMATE, 10.; SYMPOSIUM ON THE URBAN ENVIRONMENT, 14., 2018, New York. Anais [...]. New York: [s. n.], 2018. p. 1-6.

TARIFA, José Roberto; ARMANI, Gustavo. As unidades climáticas urbanas da cidade de São Paulo: os climas e a (re) produção do espaço nas metrópoles. In: ENCUENTRO DE 
GEÓGRAFOS DE AMÉRICA LATINA, 8., 2001, Santiago. Anais [...]. Santiago: USC, 2001. p. 1-12.

UNFPA. Fundo de Populações das Nações Unidas. Caderno de Populações. Disponível em: < https://brazil.unfpa.org/sites/default/files/pub-pdf/caderno_populacao6.pdf>. Acesso em: 15 ago. 2015.

$X U$, Young et al. A cotraining approach to the classification of local climate zones with multisource data. In: IEEE INTERNATIONAL GEOSCIENCE AND REMOTE SENSING SYMPOSIUM, 2017. [S. I.]. Anais [...]. [S. I.: s. n.], 2017. p. 1209-1212.

WANG, Ran et al. Mapping the local climate zones of urban areas by GIS-based and WUDAPT methods: A case study of Hong Kong. Urban Climate, [S. I, v. 24, p. 567-576, june 2018.

ZHONGLI, Lin; HANQIU, Xu. A study of urban heat island intensity based on "local climate zones": A case study in Fuzhou, China. In: INTERNATIONAL WORKSHOP ON EARTH OBSERVATION AND REMOTE SENSING APPLICATIONS (EORSA), 4., 2016, Guangzhou. Anais [...]. Guangzhou: IEEE, 2016. p. 250-254.

Recebido: julho de 2020. Aceito: março de 2021. 